\title{
LA ACCIÓN Y EL ENFOQUE PSICOSOCIAL DE LA INTERVENCIÓN EN CONTEXTOS SOCIALES: ¿PODEMOS PASAR DE LA MODA A LA PRECISIÓN TEÓRICA, EPISTEMOLÓGICA Y METODOLÓGICA?.
}

\author{
THE ACTION AND THE PSYCHOSOCIAL APPROACH OF THE INTERVENTION \\ IN SOCIAL CONTEXTS: CAN WE MOVE FROM THE FASHION TO THE THEORETICAL, \\ EPISTEMOLOGICAL, AND METHODOLOGICAL PRECISION?.
}

Recibido: octubre de 2011 - Revisado: Marzo de 2012 - Aceptado: 30 de Mayo de 2012

Por: Juan David Villa Gómez

\section{RESUMEN:}

El siguiente artículo derivado de investigación, se plantea la pregunta en torno a una realidad que viene creciendo en Colombia: el incremento de los proyectos de intervención psicosocial en diversos sectores sociales, especialmente con víctimas del conflicto armado, violencia social, violencia de género e intrafamiliar, entre otras; además de población en situación de pobreza extrema, desplazamiento forzado y víctimas de catástrofes naturales. En relación con esta lógica de intervención y de acción, la reflexión académica aún sigue siendo escasa, poco clara y repetitiva de concepciones y disquisiciones internas de las disciplinas sociales, especialmente de la psicología. Por lo tanto, desde la experiencia de trabajo, desde la propia investigación y desde los procesos de acompañamiento psicosocial desarrollados por cerca de 15 años, el autor presenta una propuesta de reflexión, que implica una mirada desde el pensamiento crítico social y una postura que permita darle un marco a la acción psicosocial en una perspectiva emancipatoria.

\section{PALABRAS CLAVES:}

Intervención psicosocial, Perspectiva psicosocial, Víctimas.

\section{ABSTRACT:}

The following article, derived from research, poses the question about a growing issue in Colombia: the increase in projects of psychosocial intervention in diverse social sectors, particularly with victims of the armed conflict, social violence, violence of gender and family, etc., as well as population in a situation of extreme poverty, forced displacement and victims of natural disasters. In relation to this logic of intervention and action, the academic reflection tends to be scarce, unclear and repetitive of concepts and internal disquisitions of the social disciplines, especially of psychology. Therefore, from the work experience, from the research itself and from the processes of psychosocial accompaniment for about 15 years, the author presents a reflective proposal, which implies a look from the social critical thought and a stance that allows to give a framework for the psychosocial action in an emancipatory perspective.

\section{KEY WORDS AND EXPRESSIONS:}

Psychosocial intervention, psychosocial perspective, victims.

${ }^{1}$ Psicólogo - Pontificia Universidad Javeriana, Magister y Doctor en Cooperación Internacional al Desarrollo Universidad Pontificia de Comillas, Madrid, España. Docente Investigador Universidad de San Buenaventura Medellin-Colombia.Juand.villa@usbmed.edu.co. 


\section{Introducción.}

Para comenzar quiero abordar una serie de preguntas que iré desarrollando a lo largo del texto. Quiero partir de un contexto en el que lo psicosocial parece haberse puesto de moda en el país, para intentar preguntarme y preguntarle al lector sobre la razones de esta coyuntura $\mathrm{y}$ en especial por las comprensiones que tenemos de lo psicosocial, puesto que empiezo a sospechar que con esta palabra puede decirse todo y nada, y a la hora de concretar los referentes, éstos no son claros, y casi que cualquier intervención comunitaria, y a veces, ni siquiera comunitaria, terapéutica y hasta psiquiátrica, siempre y cuando se haga con un grupo social, que generalmente es vulnerable, es calificada con este término.

En el contexto actual de Colombia y en la coyuntura sociopolitica del presente se ha venido incrementando desde 2005/2006 la demanda de los servicios de "atención", "intervención", "acompañamiento" psicosocial en el país. Esto puede tener varias razones:

1. En primer lugar, y lo más importante, la visibilización que las víctimas de violencia política han alcanzado en el escenario social y político nacional, quienes a través de la movilización social, las organizaciones locales, los movimientos de carácter regional, departamental y nacional se han convertido en actoras sociales y politicas relevantes, además de ser un referente con el que se debe contar a la hora de desarrollar cualquier propuesta que conduzca hacia las solución política negociada del conflicto armado, la construcción de la paz, la protección de los derechos humanos, las posibilidades de la justicia transicional, los procesos de construcción de la memoria histórica y las propuestas de reconciliación y convivencia, tanto a nivel nacional, como a nivel regional y local. Estas víctimas han demandado del Estado políticas efectivas de atención psicosocial tal como se reconoce en la sentencia T-45 de 2010 de la corte constitucional ${ }^{2}$.

2. El marco de apertura que ha posibilitado el Estado, más que por vocación o iniciativa propia, a instancias de la presión ejercida por el movimiento de víctimas en Colombia, con sus diversas expresiones y manifestaciones, pero también por la presión internacional ejercida luego de la denominada "desmovilización" de los grupos paramilitares; donde primó la atención, protección, garantías, servicios sociales y apoyos económicos a los combatientes; mientras las víctimas quedaban en un segundo plano, con todos sus derechos vulnerados. Y por la apertura cada vez mayor a analizar problemáticas complejas: violencia intrafamiliar, violencias sociales, violencias de género, procesos de deserción escolar; temas como el suicidio y el abuso sexual entre otros que, definitivamente, no pueden ser analizados desde una perspectiva monolitica; pero donde lo psicosocial no aparece con claridad metodológica ni epistemológica. Esta apertura del Estado se ha visto incrementada por el marco legal creado con la ley 1448 de 2011, conocida como ley de víctimas, que supone la generación de un programa nacional de atención psicosocial a las víctimas del conflicto armado.

3. Cada vez más ONG aparecen en el escenario nacional preocupadas por desarrollar acciones psicosociales. Algunas de reciente creación y otras de vieja data; algunas dedicadas prioritariamente al tema, otras que han estado más en la línea de la defensa de los derechos humanos o la promoción del desarrollo humano, que se han visto en la necesidad de conformar equipos al interior de sus instituciones para abordar este tipo de acciones. En

350

AGO.USB Medellín-Colombia V. $12 \quad N^{\circ} 2$ PP. 214- 547 Julio-Diciembre 2012 ISSN: 1657-8031 
muchos casos respondiendo a las demandas de intervención que se han suscitado en el Estado y en la sociedad.

4. Finalmente podría decirse que el tema se ha puesto de moda. Casi que no se concibe proyecto de intervención social o comunitaria que no incluya en alguna de sus secciones, líneas, fases o procesos la palabra psicosocial como parte de la justificación, los objetivos, los resultados o las actividades.

La primera pregunta que me hago es: ¿Por qué se ha puesto de moda? Puesto que considero que los puntos anteriores más que ser explicativos, son descriptivos de un estado de cosas. Hace 15 o 20 años, cuando se iniciaron las acciones psicosociales en Colombia, en el marco del conflicto social, político y armado, entre otras problemáticas complejas en el país; a tientas, algunas instituciones (ONG, algunas facultades de psicología) empezaron a realizar propuestas de una acción psicosocial para afrontar las consecuencias que dejaba este conflicto a lo largo y ancho del país. Se desarrollaron además algunas acciones que en el campo de la educación y el fortalecimiento comunitario tuvieron aportes desde el enfoque. Sin embargo, eran intervenciones marginales, por fuera de los centros del poder / saber y en muchos casos en contravia de estos mismos centros.

Al interior de muchas instituciones, especialmente ONG de derechos humanos o de promoción del desarrollo humano, se consideraba que: lo menos que necesitaba el país en la coyuntura que vivía, era la intervención de psicólogos. Yo mismo asistí a esa discusión, en una institución muy prestigiosa de este país, cuando se cuestionaba de manera vehemente la elaboración de un proyecto de "acompañamiento" psicosocial a población desplazada y retornante en el Bajo Atrato.

Precisamente, estas discusiones, en mi concepto, fueron las que promovieron la generación de organizaciones no gubernamentales dedicadas con prioridad al trabajo psicosocial, puesto que quienes iban comprendiendo la importancia y la necesidad de un enfoque psicosocial de la intervención comunitaria y el diseño de estrategias psicosociales para el acompañamiento de las personas y comunidades, sujetos individuales y colectivos afectados por el conflicto en el país, las comunidades afectadas por desastres naturales, o la experiencia misma de problemáticas complejas de raigambres social y cultural, tuvieron que desarrollar sus proyectos en contravía a la práctica "normal" de la disciplina (psicología y psicología social), pero también en contravia a la forma de comprender la intervención social y comunitaria desde otros marcos disciplinares: ¿Qué ha pasado de allá a estos tiempos en que lo psicosocial se ha puesto de moda? Creo que es necesario realizar una revisión y una investigación que permita comprender esta dinámica y este proceso. Sin embargo, la tarea de este artículo implica generar algunas preguntas y reflexiones al respecto.

Lo cierto es que nos encontramos con un contexto social y politico, donde las demandas del Estado, de las organizaciones sociales de base y de la cooperación internacional le están dando prioridad al trabajo psicosocial como una forma de abordar algunas problemáticas, tanto de las víctimas de violencia política, como de los excombatientes (reales o imaginarios), de los y las participantes en situaciones de violencia social, familiar o de género. Pero también para el empoderamiento de jóvenes, comunidades, mujeres, niños y niñas, entre 
otros, como actores sociales y políticos de transformación social; o simplemente como beneficiarios de acciones del Estado que llevan el rótulo de "psicosocial". Ahora bien, no me queda tan claro si la pregunta y la demanda se realiza desde las mismas comunidades, las sociedades locales, e incluso, desde la sociedad nacional, o si responde a una forma de abordar las problemáticas que al ganar terreno en algunos espacios del saber / poder, y visibilizarse desde el Estado, se observa la necesidad de dichas acciones.

\section{Metodologia.}

Me parece pertinente, volver a preguntar: ¿De qué hablamos cuando hablamos de los psicosocial? Esta es una pregunta importante, porque implica una reflexión seria que implique lo conceptual, la praxis, lo epistémico y la concepción de lo humano. Considero que no toda acción de las que está siendo denominada en la actualidad con el apelativo de lo psicosocial puede ser identificada como tal; puesto que no tienen claridad en realizar un ejercicio donde se aborda ese lugar bisagra entre lo estructural social y lo individual (Martín Baró, 1983, 1990), o bien porque no se reconoce lo subjetivo como un proceso emergente de la interacción social, simbólica y comunicativa (Mead, 1932; Carmona, 2009). Porque no aborda al sujeto en contexto ni al contexto con sus sujetos, de tal manera que o bien se desarrollan acciones individuales en contextos comunitarios, o bien se desarrollan acciones grupales que siguen teniendo como fondo una ontología y una epistemología individualista y dualista de tipo cartesiano.

Por ejemplo, en la trayectoria de trabajo que he desarrollado durante 15 años en diversas regiones del país, me he encontrado con intervenciones comunitarias, basadas en talleres y múltiples actividades que se ligan de forma superpuesta, donde no existe un diálogo entre los diversos profesionales, donde no se logra ni la interdisciplinariedad ni la transdisciplinariedad; donde simplemente cada actor cumple con sus funciones y no logra generar una interacción; donde los proyectos tienen metas y resultados en el corto plazo, centrados en indicadores numéricos que no valoran el proceso y la misma experiencia que se vive, donde se cumple con una actividad como parte de un cronograma y donde la visión se centra en la ejecución de una planeación y unos recursos, en estos casos puede plantearse la pregunta por el enfoque y la perspectiva psicosocial de su acción.

Algunos procesos basados o bien, en proyectos de atención humanitaria a población vulnerable, o programas sociales de vivienda, generación de ingresos, entre otros, que ligan de forma superpuesta lo individual y lo grupal, que no dialogan con la gente, no toman en consideración los saberes de la población con la que trabajan y la toman simplemente como "beneficiaria" o "usuaria" de un servicio, desde un marco de actuación asistencial, aunque en el papel se diga lo contrario; que en muchos casos, generan dependencias, pasividad y pérdida de poder en la gente, desarrollan intervenciones que no implican una mirada psicosocial, un enfoque psicosocial.

De otro lado, también es común del lado de las ciencias "psi" encontrarse con intervenciones, donde se está tratando a algunas víctimas de violencia social o política con medicamentos psiquiátricos, a partir de diagnósticos patologizantes y psicologistas construidos con base en las nosologías de la psicopatología, desconociendo en muchos casos los contextos sociales,

352

AGO.USB Medellín-Colombia $\quad$ V. $12 \quad N^{\circ} 2$ PP. 214- 547 Julio-Diciembre 2012 ISSN: 1657-8031 
relacionales, organizativos en los que se mueven las personas y que, por lo tanto, generan inmovilidad, retiro de lo público y una carga más en los sujetos (Summerfield, 2000, 2001; Lykes, 2001). En mi concepto estas actuaciones psicologistas no pueden ser avaladas como psicosociales.

\section{Resultados - discusiones.}

¿Qué entendemos por lo psicosocial?.

En este contexto en que pululan los proyectos "psicosociales" me parece urgente determinar a qué nos referimos cuando hablamos de lo psicosocial. Y creo que es fundamental que desde la academia, y en un espacio como el de la revista El Ágora, nos pongamos en la tarea de una discusión más profunda sobre lo que significa: ¿Es una perspectiva o es un enfoque? ¿Es una metodología o es un marco epistemológico? ¿Es una disciplina que se equipara a la psicología social o es un marco de intervención de las ciencias "psi”? ¿Se corresponde a un marco disciplinar o es una visión transdisciplinar que posibilita una mirada que puede permear la acción de otras disciplinas? ¿O puede ser todas estas cosas a la vez? Me parece que la discusión no es trivial, porque de alli parten las formas, las maneras, las líneas de acción, los programas y proyectos. Y una mirada, una forma de enfocar un proyecto generará unos u otros resultados en los procesos sociales que se acompañan. Y aquí vuelve y cabe la pregunta: ¿Se acompaña, se interviene, se atiende? Quizás la palabra acompañamiento, en mi concepto, tenga más cercanías con lo que puede entenderse por psicosocial.

Para comenzar vale decir que una perspectiva y/o un enfoque psicosocial incluyen principios como los de dignidad, apoyo mutuo, solidaridad, vida con calidad, enfoque de derechos, enfoque de género y desarrollo humano integral en salud mental. Es fundamental que todos estos marcos de acción hagan parte de los procesos que tienen el apelativo. Esto implica, a su vez, una acción centrada en la promoción de prácticas relacionales basadas en el reconocimiento y la valoración del ser humano en ejercicio de construcción. Todos estos elementos interactúan sistémicamente y son fundamentales a la hora de comprender y abordar lo psicosocial (González \& Villa, 2012). Hablar de acompañamiento psicosocial implica, por tanto, una posición que atraviesa no solo lo teórico y la intervención misma, sino también un lugar ontológico, ético-político, epistemológico y metodológico:

A nivel ontológico está implicada una concepción del ser humano como sujeto en relación y en construcción con otros y otras; el cual es constituido por condiciones biológicas, psicológicas, histórico-sociales, culturales, económicas, políticas que lo definen, en un proceso sistémico de interacción social, comunicativa y simbólica que implica la emergencia de la propia subjetividad personal y la construcción y/o reconstrucción de la colectividad (Carmona, 2009). No es un individuo, en el sentido solipsista de la palabra. Es decir, lo que cada persona es, lo es en relación e interacción continua con los otros y otras, y con su mundo simbólico. Implica además una concepción de la realidad que va más allá de lo material y que supera las visiones funcionalistas y mecanicistas, donde lo relacional, lo narrativo, el lenguaje (sus usos y significados en el marco de la pragmática), lo simbólico y lo cultural hacen parte de los elementos sistémicos que entran a formar parte del análisis de lo real en lo humano (Vásquez, 2001). 
En términos epistemológicos implica una opción que privilegia una mirada en la que el sujeto que conoce e interviene no debe ser el "ajeno" o "extraño", que no se separa y se asume de manera "neutral" y "objetiva" frente a la realidad que acompaña; puesto que o bien, hace parte de esa realidad, o bien, debe "insertarse" en el marco de esa realidad para comprenderla desde adentro, desde sus propias determinaciones y actuar, conocer, investigar e interactuar desde las categorías, saberes y dinámicas de ese espacio social, que implica a unos sujetos y a una colectividad (Martín Baró, 1985; Martín Beristain y Doná, 1997; Martín Beristain, et. al, 1999). Implica también una concepción de la realidad, en la cual ésta no está separada del sujeto; es decir, se concibe una interacción profunda entre sujeto y realidad, al punto que ésta no puede ser concebida como independiente del mismo. Para decirlo a la manera del teorema de Thomas: "Si los seres humanos definen una situación como real, ésta es real en sus consecuencias" (Carmona, 2009, 2012).

Al analizar las interacciones y las relaciones sociales, no se pierde de vista el marco sociopolítico e histórico como un todo, dentro del cual se construyen y desarrollan los sujetos, constituidos como tales en los procesos de interacción social y simbólica. Por lo tanto a la hora de observar, diagnosticar, analizar y evaluar se tienen en cuenta no solo sintomatologías o trastornos, procesos epidemiológicos, patologías sociales y carencias; sino también procesos colectivos, significados y sentidos, afrontamientos y factores de resiliencia individual y comunitaria. Procesos de reivindicación social, luchas sociales y políticas que constituyen a sujetos empoderados, seres humanos en proceso de construcción y de afirmación de su propia identidad, es decir, sanos, vivientes, sobrevivientes, que a pesar, incluso de algunos sintomas, tienen la capacidad de sobreponerse al dolor y al sufrimiento, para afrontarlo, transformarlo y transformarse a sí mismos y a la sociedad en que habitan.

A nivel metodológico en el plano de la investigación se incluyen métodos cualitativos como procedimientos para conocer la realidad, para realizar aproximaciones a esa realidad compleja, sin que se aspire a una verdad como adecuación, ni a la construcción de leyes y teorias universales explicativas de los fenómenos, sino a interpretaciones densas de los mismos (Geertz, 1989). En términos de intervención se concibe que estrategias de acompañamiento y acción relacionales (familiares, colectivas/comunitarias y públicas) pueden tener una incidencia más profunda sobre la construcción y la transformación de la subjetividad de quienes participan en las mismas; lo cual, implica que se privilegien intervenciones grupales, comunitarias, colectivas y públicas, sobre las individuales, aunque estas últimas no son excluidas del enfoque.

A nivel ético-político está implicada una opción fundamental por el otro, la persona vulnerable, la víctima, el excluido, el invisible y silenciado, por las mayorias de nuestra población, como afirmaba Martín-Baró (1983, 1985), puesto que nos implicamos en una psicología situada, desarrollada desde contextos específicos, respondiendo a las demandas, necesidades y procesos de nuestro país y nuestro continente latinoamericano, tanto desde una perspectiva teórica, académica, con rigor científico; pero al mismo tiempo comprometida con las realidades de nuestros contextos. 
Por esta razón, puede afirmarse con claridad que la patologización o el análisis simplista e individual de los sujetos que deben vivir situaciones limite, son una forma de reproducir los sistemas de exclusión, dominación, explotación y victimización que se generan en nuestras sociedades; puesto que atribuye la responsabilidad de las consecuencias de los hechos violentos y/o de exclusión a las personas, al nombrarse como enfermedad, problema psicológico, trastorno, malestar individual, patología social, anormalidad, etc. (Martín Baró, 1985; Summerfield, 2000, 2001; Lykes, 2001; Martín Beristain, 2005). Lo cual termina siendo un problema individual e interno del sujeto, y no un producto de procesos complejos de interacción social, política e histórica (Carmona, 2009) a partir de situaciones estructurales de exclusión, procesos de acumulación de poder, dinámicas sociales, políticas y económicas excluyentes o graves violaciones a los derechos humanos en el contexto de un Estado social de derecho.

En esta línea, el enfoque y la perspectiva psicosocial se presentan como la construcción de un modelo de intervención e investigación que reclama en los psicólogos, pero también en otras profesiones que están siendo convocadas a los proyectos de "intervención" que se atribuyen esta denominación, una perspectiva transdisciplinar de la propia disciplina que les posibilite un diálogo abierto y riguroso con profesionales y científicos de diversas áreas; así como de esfuerzos cada vez más articulados, a manera de procesos que involucran a las personas, a las comunidades, a la sociedad y al Estado.

Desde allí se abren marcos de comprensión y transformación de situaciones problemáticas que inciden en la salud mental de los habitantes de la ciudad, del departamento y del país, y que son cada vez más urgentes de comprender e intervenir, como la violencia política, las violencias de género, la violencia sexual, la violencia social y la violencia intrafamiliar, entre otras; los problemas de exclusión social y empobrecimiento, el pobre desarrollo humano y los bajos estándares de bienestar en nuestra población; la construcción de subjetividades e identidades sociales y políticas en contextos múltiples y muchas más problemáticas en las que los psicólogos y estos profesionales están llamados a actuar.

Nuestra realidad local y nacional, con problemáticas psicosociales tan arraigadas desde el contexto histórico, evidencia la necesidad de personal humano idóneo, que cuente con las competencias necesarias para hacer un acercamiento proactivo a las situaciones y problemáticas psicosociales, capaz de adelantar intervenciones integrales, sistémicas y de amplio impacto, con una visión inter y transdisciplinaria. Por esta razón es necesario y pertinente una reflexión profunda de lo que significa hacer intervención desde un enfoque psicosocial con el fin de desarrollar habilidades, competencias y conocimientos que posibiliten actuar en estos escenarios diversos, no solamente desde visiones teóricas o paradigmáticas, sino que lleven a la implicación e inclusión como actores transformadores dentro de estos procesos sociales.

Esta reflexión me lleva también a tener presente la relación que se viene estableciendo entre los psicosocial y lo jurídico, en un marco de protección de derechos, búsqueda de reparaciones y valoración del daño que se ha sufrido, con el fin de buscar medidas de restitución, rehabilitación y reparación. Porque aquí, si bien, el problema es en otro ámbito, la situación puede ser similar. Puesto que resulta que a la hora de valorar el daño, el discurso 
jurídico, que tiende a ser un discurso "positivo", que exige pruebas y hechos, necesita referentes que puedan ser reconocidos en una corte, e una instancia judicial. Así pues, cuando se hace la valoración, tiende a primar una mirada que pueda contar con un reconocimiento explícito de la "ciencia normal", de la terminología psiquiátrica o psicopatológica, que permita medir, observar, cuantificar, determinar el daño, y esto se hace en términos de sintomas, trastornos, daño psíquico y demás usos nosológicos. Y, por otro, lado, lo social, lo cultural, se valora, generalmente, de forma escindida desde otros parámetros de la ciencia social. Y en mi concepto, lo propiamente psicosocial, termina diluyéndose.

Por lo tanto, cabe preguntarse nuevamente si cualquier intervención o proceso de acompañamiento que realiza actividades colectivas, comunitarias, es un acompañamiento psicosocial. ¿Cualquier acción comunitaria: talleres, dinámicas, ejercicios colectivos, sociodramas, etc. porque constituyen metodologías de trabajo comunitario, o de educación popular, por el simple hecho de serlo, son una acción psicosocial? Esto nos pone en relación con una nueva pregunta: "¿Cuáles son las claves a tener en cuenta en los procesos de acompañamiento psicosocial?” Creo que es una pregunta pertinente y necesaria.

Una perspectiva de la actuación psicosocial.

¿La reflexión que estoy proponiendo implicaría, entonces, que existe una única versión de lo psicosocial, y un modelo verdadero, mientras los otros son falsos? Más allá de una mirada desde el lugar de la verdad, creo que es fundamental que se pueda vislumbrar lo que implica la acción y el acompañamiento psicosocial, puesto que más allá de la acción concreta, creo que cuando hablamos de lo psicosocial, nos referimos más a un enfoque, a una epistemología, a una forma de comprender lo humano (una ontología del sujeto humano), que deriva en unos métodos particulares, donde lo relacional y lo vincular, lo contextual y la interacción son fundamentales.

En primer lugar, esta mirada no implica descartar la afectación del mundo emocional o psicológico y la necesidad de actuar sobre este nivel, ya que la violencia padecida y otras experiencias límite afectan todo el mundo psíquico; sin embargo, el enfoque psicosocial, avanza hacia una mirada que pretende el fortalecimiento y la reconstrucción del tejido social, entendiendo que las reacciones emocionales no son un suceso aislado del mundo relacional y social. Abordar sólo lo psíquico implicaría desconocer que en este tipo de situaciones hay un contexto social y político que está afectando y es, además, constitutivo de lo subjetivo, un contexto de injusticia y deshumanización en el que se necesitan cambios sustanciales; lo que equivale a individualizar el sufrimiento que, en este tipo de contextos, resulta más funcional para los intereses de quienes victimizan (Summerfield, 2000, 2001; Lykes, 2001; Das, 2008).

Un enfoque psicosocial implicará considerar la particularidad de la población vulnerable, víctima o que se encuentra en la experiencia de un sufrimiento, para con ello realizar el reconocimiento de los múltiples contextos sociales, políticos, culturales en los cuales están insertos para hacer una intervención respetuosa con estas dimensiones incorporando, necesariamente, estos elementos para generar un proceso de acompañamiento integral. 
Así pues, se elige no mirar a las personas como entes separados (individuos), sino en relaciones con su entorno vital, con los otros, con la comunidad, y a su vez mirar la comunidad como un todo que se relaciona con su entorno contextual, regional, con otras comunidades y con sus miembros individuales. La mirada, es decir la forma de percibir, comprender e interpretar la realidad, (el paradigma si se quiere) se centra en otro lugar: el lugar del sujeto en construcción permanente en su interacción con la colectividad que está sujeta a vicisitudes, situaciones límite, experiencias de ruptura, procesos de exclusión, violación de derechos, relaciones de dominación, etc. Es decir, no ha logrado fortalecer sus procesos colectivos en la búsqueda de su desarrollo integral y sostenible; ha sido explotado o sistemáticamente excluido, o su comunidad ha sido golpeada para romper su tejido, su organización y sus lazos de solidaridad, para des-dignificar el sentido vital de las personas, las familias, la comunidad en general.

Y es desde alli que se le apuesta a encontrar esos sentidos, esos espacios de solidaridad, a construir conjuntamente estrategias para mantenerse y no perder la dignidad aún en las peores circunstancias, para reconstruir y fortalecer su organización. Y esto permite asumir la realidad como actores sociales y políticos, generando procesos de recuperación de la memoria colectiva/histórica, identificando los referentes que los constituyen como tales, las historias y testimonios que dan sentido a la colectividad, pero también a las personas; la recuperación de la dignidad, a través del empoderamiento personal y colectivo, que implica procesos para desarrollar estrategias que fortalezcan el afrontamiento y la resiliencia en los avatares de las diversas situaciones límite (Martín-Baró, 1985; Martín Beristain, 2005, 2008; Villa, et al, 2007; Villa, 2009).

El fundamento de esta posición se cifra en el hecho de la construcción de la identidad, del yo, de la persona, a través de la interacción con los otros, en lo cual está implicada la cultura, el lenguaje, las normas y los valores. La forma como se construyen las relaciones en una sociedad, en una comunidad humana, va a implicar el tipo de personas que viven en esa sociedad. Así pues, la intervención y el trabajo que enfatiza la acción comunitaria va a determinar nuevas relaciones y por lo tanto transformaciones en las personas, de la misma manera que una desestructuración de estas relaciones implican desestructuraciones de la persona.

Así pues, se puede entender que este enfoque de la acción, que también tiene una dimensión trans e interdisciplinar, invita a un acompañamiento que involucre de forma interactiva, compleja y simultánea, varios ámbitos y niveles de la realidad, para que la acción resulte medianamente coherente con la complejidad de esa realidad, tal como se presenta en el modelo gráfico: un nivel subjetivo (donde se desarrollan estrategias narrativas y terapéuticas); un nivel interpersonal (familiar, grupal y comunitario) donde se desarrollan estrategias conversacionales de apoyo mutuo, memoria compartida y talleres grupales; donde se pone en evidencia el mundo de la interacción cotidiana, las construcciones grupales y el compartir social en lo cotidiano (Cfr. Villa, 2012).

Un nivel sociopolitico, donde se busca el empoderamiento de los sujetos como ciudadanos y actores de derechos, acciones públicas y simbólicas de memoria, es decir, una dimensión colectiva de la memoria que abordará las narrativas y acciones públicas que implican las 
comunidades y las sociedades en tanto estructuras que desarrollan productos simbólicos, herramientas comunicativas y culturales que están en un orden sistémico diferente al individual y al grupal; y finalmente, un nivel histórico cultural que permite posicionar los relatos, narraciones y acciones públicas de la gente en dispositivos educativos y comunicativos que recoge las narrativas, simbolos y herramientas culturales que se transmiten de generación en generación y que encuentran espacios de difusión en medios de comunicación y en la escuela, contribuyendo a la construcción de imaginarios sociales y memoria histórica, en la formación de las nuevas generaciones (Cfr. Villa, 2012).

Esta mirada implica por lo tanto un cambio de paradigma y un acercamiento diferente a la manera de comprender y conceptualizar la acción y la intervención psicosocial puesto que deja de ser un asunto de la mente, como esencia, y se transforma en un proceso dinámico que circula dentro y fuera del sujeto individual, tal como puede observarse en el siguiente esquema: 


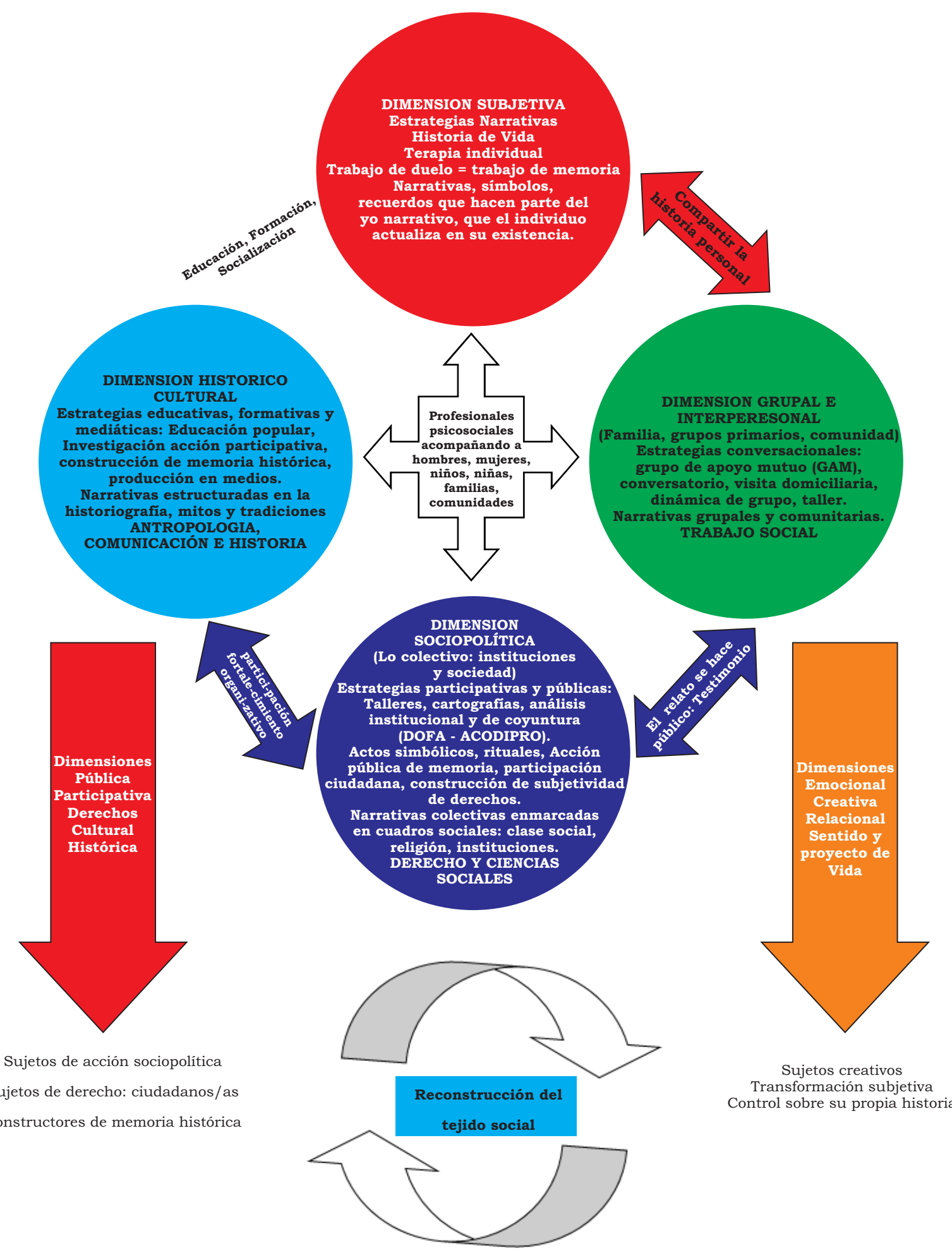


¿Qué significa este esquema?

En términos teóricos, que la intervención individual, la terapia, la narrativa y la construcción de la memoria individual y autobiográfica, son acciones centradas en la subjetividad como proceso emergente de la interacción comunicativa y simbólica. Este proceso implica en la actuación, la construcción de testimonios, narrativas personales e historias de vida que tengan una dimensión, al mismo tiempo que curativa, ejemplar; de tal manera que el trabajo personal, subjetivo, redundará en un beneficio social y el trabajo de duelo será trabajo de memoria (Ricoeur, 2003; Lira, 2011).

Cuando estos relatos personales son compartidos en un espacio grupal, toman la dimensión de una acción comunitaria. Esto implica acciones psicosociales que se constituyen en las conversaciones cotidianas que se dan en los grupos familiares, grupos primarios, donde se da el compartir social de las diversas experiencias. Este proceso es el que se da también en los grupos de apoyo mutuo -GAM (Martín Beristain, 2005, 2009; González \& Villa, 2012), los espacios de diálogo de la pedagogía de Paulo Freire (Martín Baró, 1985) son espacios donde el recuerdo, la simbolización y la expresión de la experiencia posibilitan la elaboración terapéutica, pero al mismo tiempo la reconstrucción de las relaciones cotidianas, de la confianza y del tejido comunitario, fortaleciendo la identidad colectiva, los sentidos de pertenencia, lo que, a su vez, favorece la participación en escenarios sociales y políticos (Villa, et. al, 2007; Uribe, 2010).

Así estos procesos conversacionales, donde emergen memorias compartidas, estos espacios grupales por la vía de la acción y la participación pública se constituyen en escenarios colectivos y públicos, donde las narrativas pasan del plano subjetivo al plano colectivo e histórico. La promoción y el desarrollo de escenarios participativos, en procesos de retroalimentación positiva con las dinámicas de fortalecimiento y reconstrucción del tejido comunitario, van conduciendo a la organización o al fortalecimiento de las organizaciones, de la participación y la conciencia de los propios derechos (Martín Beristain, 2005; Cfr. Villa, 2012; González y Villa, 2012).

Se entra al escenario de lo público, se desarrollan procesos colectivos y sociales, que tienen, a su vez, una dimensión política; que si logran cristalizarse desarrollan procesos de incidencia social, política para la transformación de las realidades. En este punto puede observarse la pertinencia de ejercicios y acciones en la lucha por los derechos, la movilización social y política, el trabajo de formación en la participación ciudadana, y la lucha social. Todo ello redunda en el bienestar de los sujetos individuales y colectivos.

Se construyen narrativas donde los relatos recogerán los procesos de interacción social y comunicativa que han permitido que la experiencia individual y las vivencias colectivas se tramiten en espacios grupales (grupos de apoyo mutuo - GAM y talleres de memoria), para la construcción de escenarios compartidos, que a su vez permiten la tramitación emocional de la experiencia límite, pero que refuerzan los vínculos, recuperan la confianza y reconstruyen los colectivos. Este es el escenario de las acciones públicas de memoria, la participación y el fortalecimiento organizativo, la movilización social y política, la reivindicación de los derechos y la lucha por la justicia. 
Escenarios donde se construyen sentidos y significados sociales que entran al espacio societal y disputan con otros discursos de poder, dominación, justificación de la violencia o de la explotación, discursos de indolencia, indiferencia o negación, tal como se desarrollan desde los centros del poder / saber y desde la llamada historia oficial (Schmuchler, 1986; Martin-Baró, 1990, Gaborit, 2006, Pastoriza, 2009; entre otros). Es decir se configuran escenarios para la defensa y la restitución de los derechos humanos, la transformación social y política. Y esto, a su vez, revierte en la transformación de los sujetos individuales, que logran empoderarse, reconocerse en su dignidad y sentirse sujetos de derechos, seres humanos con integridad.

En estos escenarios las comunidades, los sujetos participantes, las víctimas se esfuerzan para que sus relatos sean oídos, escuchados y constituyan referentes válidos para la cristalización de su mirada como una perspectiva, que busca, a partir de testimonios, las acciones performativas, marchas, incidencia política, reivindicaciones colectivas, trabajo comunitario y otras acciones públicas que se dé un proceso de transformación del sí mismo colectivo, pero también en la sociedad y en el Estado.

Ahora bien, este proceso pasa por un nivel que implica acciones sociales con una vocación intergeneracional y que implica escenarios que cuenten con un aval institucional y social de mayor alcance, finalmente, la dimensión histórica y cultural. Pues, esto implica que se cristalizan y se materializan en una sociedad concreta, relatos, procesos y transformaciones que se convierten a su vez en referentes de construcción de nuevos sujetos individuales y colectivos. Es decir, un proceso de intervención psicosocial integral comienza a interactuar con las experiencias y vivencias de un sujeto individual, se convierte en marco de interpretación de la realidad de ese sujeto, que es introducido en esa cultura o sociedad, en las conversaciones y relaciones interpersonales y grupales. En un proceso dialéctico que se da permanentemente y que permite, tanto la permanencia de esas narrativas, como la actualización de las mismas en cada nuevo círculo, en el que el sujeto se va apropiando de sus determinaciones culturales, pero también con el poder de irlas transformando en su propia acción.

\section{Conclusiones.}

Arribamos a un concepto de acción psicosocial abierto, en el cual ésta, como acción humana, es portadora de significados, sentidos, marcos y esquemas de interpretación de la realidad de un sujeto humano, cuando intenta construir, a partir de estos referentes una visión y una acción en el presente, para transformarlo y construir el propio futuro y el de las nuevas generaciones. Esto implica la construcción de esquemas que le permitan dirigir su acción en esa realidad, en esa sociedad. Estos marcos y esquemas interpretativos se encarnan en narrativas, simbolos, herramientas culturales, representaciones, obras de arte, discursos, conmemoraciones, acciones públicas, relatos que circulan en el mundo social y que están disponibles, tanto en el habla y la historia contada por los sujetos, como en la interacción comunicativa en sus grupos de referencia y en las producciones sociales, políticas y culturales que se convierten en discurso y acción pública que también puede ser analizada, evaluada y estudiada. 
Por lo tanto, estos ámbitos, implican unos modos de actuación que, invitan a un acompañamiento que involucre este proceso interactivo y complejo en varios órdenes de la acción:

- Acciones y estrategias para la restitución emocional (que pueden ser individuales o colectivas).

- Apoyo a la gente en los procesos de reconstrucción organizativa que impliquen el fortalecimiento de los colectivos, de la movilización pública y recuperación de sentidos de pertenencia.

- Fortalecimiento de la participación ciudadana, que posibilite un ejercicio de interlocución con las múltiples instancias del Estado.

- Apertura de espacios para la lucha y la reivindicación de los derechos, que posibiliten una visión de sí mismos / as como sujetos de derecho y el ejercicio de una ciudadanía plena.

- Para las dos anteriores, será fundamental trabajar en la conciencia histórica de los sujetos, los procesos de memoria social y colectiva; además de la construcción de identidades sociales incluyentes que conduzcan al respeto por la diferencia, la justicia y la equidad.

- Reconstrucción de marcos, tradiciones, costumbres y referentes culturales que posibiliten una valoración de la propia cultura.

- Construcción de subjetividades solidarias para la generación de procesos económicos colectivos y cooperativos que permitan trasformar relaciones de explotación e inequidad estructural.

- Formación política para la concientización y la acción en procesos de construcción de lo público y del Estado.

Por lo tanto, más allá de las actividades concretas, de los talleres y de las intervenciones grupales. Más allá de una acción sobre malestares y sintomas subjetivos, más allá de la terapia, la acción psicosocial se comprende como una mirada, una perspectiva y, al mismo tiempo, una forma de enfocar la realidad que posibilita escenarios de actuación que comprenden los fenómenos humanos desde una visión integral y que por lo tanto, plantean alternativas, soluciones y procesos incluyentes que se despliegan en el tiempo y que producen nuevas subjetividades en marcos de mayor justicia, equidad, desarrollo a escala humana, sana convivencia y paz. 


\section{Referencias.}

Carmona, J. (2009) La carrera de las niñas soldado en Colombia: un estudio desde el punto de vista del agente. Tesis Doctoral. Departamento de Psicología, Universidad Complutense, Servicio de Publicaciones, Madrid.

Carmona, J. (2012) ¿Qué es lo psicosocial? Cinco principios de respuesta a la pregunta. Ponencia presentada en el seminario “¿Qué es lo Psicosocial?”, abril de 2012, Fundación Universitaria Luis Amigó, Medellín.

Das, V. (2008) La Antropología del Dolor. En: Ortega, F. (Ed.) Veena Das: Sujetos del dolor, agentes de dignidad. PP. 409 - 436. Pontificia Universidad Javeriana y Universidad Nacional de Colombia, Bogotá.

Geertz, C. (1989). La interpretación de las culturas. Ed. Gedisa, Barcelona, España.

Gergen, K. (1996) Realidades y Relaciones. Aproximaciones a la Construcción Social. Ed. Paidós, Barcelona.

González, P. \& Villa, J.D. (2012) Elementos para la intervención psicosocial y en salud mental a victimas de violencia politica en Antioquia. Una propuesta para el personal psicosocial. Gobernación de Antioquia, Medellín.

Lykes, M. B. (2001) A critical re-reading of PTSD fron a cross-cultural community perspective. En: Hook, D. and Eagle, G. (Eds.) Psychopatology and social predjudice. PP. $92-108$. UCT pres / JTA, Cape Town, South Africa.

Lira, E. (2011) Las Victimas Testigos Históricos Sujetos de Justicia El testimonio de experiencias politicas traumáticas: terapia, denuncia y memoria. Cátedra internacional Martín-Baró, Pontificia Universidad Javeriana, Bogotá, Colombia.

Martín-Baró, I. (1983) Acción e Ideología: Psicología social desde Centroamérica I. UCA Editores, San Salvador.

Martín-Baró, I. (1985) Hacia una psicología de la liberación. En: Blanco, A, (ed.) (1991) Psicología de la liberación. Ed. Trotta, Madrid, pp. 283-302.

Martín-Baró, I. (1990). Guerra y Salud Mental. En Psicología Social de la Guerra. San Salvador: UCA editores.

Martin Beristain, C. et. al. (1999) Reconstruir el tejido social. Icaria. Barcelona.

Martin Beristain, C. (2005) Procesos de duelo en las comunidades mayas afectadas por violencia politica. Tesis en psicología social. Facultad de Psicología, Universidad del País Vasco, Bilbao. 
Martín Beristain, C. (2009) Diálogos sobre la reparación. Qué reparar en los casos de violaciones de derechos humanos. Ministerio de Justicia y Derechos Humanos. Quito, Ecuador.

Martín Beristain, C. \& Doná, G (1997) "Enfoque Psicosocial de la ayuda humanitaria". Universidad de Deusto, Instituto de Derechos Humanos. Bilbao.

Mead, G. (1932 / 1983) Espiritu, Persona y sociedad. Ed. Paidós, Buenos Aires.

Pastoriza, L. (2009) Hablar de memorias en Argentina. En: Vinyes, R. (Ed.) El Estado y la memoria: Gobiernos y ciudadanos frente a los traumas de la historia. PP. 291 239. Memorial Democratic, RBA Libros, Barcelona.

Ricoeur, P. (2003) La Memoria, la historia, el olvido. Ed. Trotta, Madrid.

Schmucler, H. (1996) Ni siquiera un rostro donde la muerte hubiera podido estampar su sello. Reflexiones sobre los desaparecidos y la memoria. En: Revista Pensamiento de los confines, No. 3, Septiembre, PP. 9-12.

Summerfield, D. (2000) War and mental health: A briefoverview. En: British Medical Journal, No. 321, PP. 232 - 235.

Summerfield, D. (2001) The invention of post-traumatic stress disorder and the social usefulness of a psychiatric category. En: British Medical Journal, No. 322, PP. 95 - 98.

Uribe, M.V. (coord.) (2010) Memoria en tiempos de guerra: repertorio de iniciativas. Investigación del grupo de memoria histórica de la Comisión Nacional de Reparación y Reconciliación. Puntoaparte Editores. Bogotá.

Vásquez, F. (2001) La memoria como acción social: Relaciones, significados e imaginario. Paidós, Barcelona.

Villa, J.D. Tejada, Carolina; Sánchez, Nathalie \& Téllez, Ana María. (2007) Nombrar lo innombrable: Reconciliación desde la perspectiva de las víctimas. CINEP, Bogotá.

Villa, J.D. (2009) La memoria como territorio en disputa y fuente de poder: un camino hacia la dignificación de las víctimas y la resistencia noviolenta. En: Briceño Donn, M. et. al. (ed.) Recordar en conflicto. Iniciativas no oficiales de memoria en Colombia. ICTJ. Bogotá.

Villa, J. D. (2012) El papel de la memoria colectiva de las organizaciones de víctimas en la reconstrucción del tejido social y empoderamiento colectivo. Tesis Doctoral, Instituto de estudio sobre las Migraciones, Universidad Pontificia de Comillas, Madrid. 
2 "La prestación de los servicios de salud a las víctimas del conflicto armado interno que además ostentan la calidad de desplazados no puede limitarse únicamente a los planes básicos que se contemplan en cada uno de los regimenes, es decir, en el Régimen Contributivo y en el Subsidiado, debido a que en el diseño de estos programas no se contemplaron las especificidades que se derivan de la condición de víctima del conflicto interno. Como lo señaló el Ministerio de la protección. Social en su intervención, estos planes no contemplan, entre otras cosas, atención psicológica y psiquiátrica de mediana y alta complejidad asi como la implementación efectiva de un enfoque psicosocial, elementos necesarios para la prestación de los servicios de salud a las victimas integralmente". 